\title{
Coexistence and Spectral Efficiency in Decentralized Networks
}

\author{
Kamyar Moshksar \\ Dept. of Electrical Engineering \\ University of Waterloo \\ Waterloo, ON, N2L 3G1
}

\author{
Alireza Baysteh \\ Dept. of Electrical Engineering \\ University of Waterloo \\ Waterloo, ON, N2L 3G1
}

\author{
Amir K. Khandani \\ Dept. of Electrical Engineering \\ University of Waterloo \\ Waterloo, ON, N2L 3G1
}

Email:kmoshksa@shannon2.uwaterloo.ca Email: alireza@shannon2.uwaterloo.ca Email: khandani@shannon2.uwaterloo.ca

\begin{abstract}
We consider a wireless communication network with a fixed number of frequency sub-bands to be shared among several transmitter-receiver pairs. In traditional frequency division (FD) systems, the available sub-bands are partitioned into disjoint clusters (frequency bands) and assigned to different users (each user transmits only in its own band). If the number of users sharing the spectrum is random, this technique may lead to inefficient spectrum utilization (a considerable fraction of the bands may remain empty most of the time). In addition, this approach inherently requires either a central network controller for frequency allocation, or cognitive radios which sense and occupy the empty bands in a dynamic fashion. These shortcomings motivate us to look for a decentralized scheme (without using cognitive radios) which allows the users to coexist, while utilizing the spectrum efficiently. We consider a frequency hopping (FH) scheme (with iid Gaussian code-books) where each user transmits over a selection of sub-bands and hops to another selection (with the same cardinality) from transmission to transmission. We derive lower and upper bounds on the achievable rate of each user and demonstrate that for large signal-to-noise ratio (SNR) values, the two bounds coincide. This observation enables us to compute the sum-rate multiplexing gain (SMG) of the system. Subsequently, we show how each user can regulate its rate to guarantee fairness while maximizing SMG. We compare the FH and FD systems in terms of the following performance measures: average sum-rate multiplexing gain $\left(\eta_{1}\right)$, average multiplexing gain per user $\left(\eta_{2}\right)$, the minimum multiplexing gain per user $\left(\eta_{3}\right)$ and service capability. We show that (depending on the probability mass function of the number of active users), the FH system can offer a significant improvement in terms of $\eta_{1}$ and $\eta_{2}$ (implying a more efficient usage of the spectrum). It is also shown that $\frac{1}{e} \leq \frac{\eta_{3}^{(F H)}}{\eta_{3}^{(F D)}} \leq 1$, i.e., the loss incurred in $\eta_{3}$ is not more than $\frac{1}{e}$. Finally, computation of the so-called service capability shows that in FH systems any number of users can coexist fairly, while the maximum number of users in FD system is limited by the number of available bands.
\end{abstract}

\section{INTRODUCTION}

Optimal resource allocation is an imperative issue in wireless networks. When multiple users share the same spectrum, the destructive effect of multi-user interference can limit the achievable rates. As such, an effective and low complexity frequency sharing strategy which maximizes the degrees of freedom per user, while mitigating the impact of the multi-user interference is desirable. In frequency division (FD) systems, different users transmit over disjoint frequency bands. Due to practical considerations, such FD systems usually rely on a fixed number of such frequency bands. The main drawback of FD systems is that most of the time the majority of the potential users may be inactive, reducing the resulting spectral efficiency. Reference [1] considers a network of several users with mutual interference. Treating the interference as noise, a central controller computes the optimum power allocation of each link over the spectrum to maximize a global utility function. This leads to the best spectrum sharing strategy for a specific number of users. Clearly, if the number of users changes, the system is not guaranteed to offer the best possible spectral efficiency. In fact, it is shown in [1] that if the crossover gains are sufficiently greater than the forward gains, the frequency division is optimum. However, as mentioned earlier, if the number of users sharing the spectrum is random, FD systems can be highly inefficient in terms of the overall spectral efficiency. To avoid the need for a central controller, cognitive radios [2] are introduced which can sense the bands and transmit over an unoccupied portion of the available spectrum. Fundamental limits of wireless networks with cognitive radios is studied in [3]. Although cognitive radios avoid the use of a central controller, they require methods for frequency sensing and dynamic frequency assignment which add to the overall system complexity. Noting the above points, it is desirable to have a decentralized frequency sharing strategy (without the need for cognitive radios) which allows the users to coexist, while utilizing the spectrum efficiently and fairly.

Motivated by the above observations, we consider a decentralized network operating on a set of $u$ frequency subbands to be shared among $n$ users. Different transmitters are linked to different receivers through paths with static and nonfrequency-selective fading. Each user is assumed to have no prior knowledge about the code-books of the other users. We propose a frequency hopping $(\mathrm{FH})$ strategy in which the $i^{t h}$ user selects $v_{i}$ frequency sub-bands among the $u$ available subbands and hops to another set of $v_{i}$ sub-bands for the next transmission. It is assumed that all users transmit independent Gaussian code-books over their chosen frequency bands.

As each user hops over different subsets of the sub-bands without informing other users about its hopping pattern, sensing the spectrum to track the instantaneous interference is a difficult task. This assumption makes the interference probability density function (PDF) on each frequency sub- 
band at the receiver side of each user be mixed Gaussian. Since the channel gains have a continuous PDF, the number of Gaussian components in the interference PDF is $2^{n-1}$ with probability one. Each user is able to derive the interference PDF after a sufficiently long training period. Being a random variable, the number of active users in the system is taken to be a global knowledge as it can be inferred from the number of interference levels.

We derive upper and lower bounds on the achievable rate of each user which coincide in the high SNR regime. This enables us to obtain the sum-rate multiplexing gain of the network. We show how each user can regulate its rate close to the achievable rate within a gap which saturates as SNR increases. In fact, the only information each transmitter needs is the highest interference level at its affiliated receiver and its forward channel gain.

We compare the FD system with the FH system based on four measures namely, average sum-rate multiplexing gain $\left(\eta_{1}\right)$, average multiplexing gain per user $\left(\eta_{2}\right)$, minimum multiplexing gain per use $\left(\eta_{3}\right)$ and service capability where the latter is the average of the fraction of users who are getting service out of the total number of active users.

We show cases (depending on the probability mass function of the number of active users) where the FH system offers larger values of $\eta_{1}$ and $\eta_{2}$ implying more efficient frequency usage. In fact, the FD system is already designed to service up to $K \leq u$ users where $K \mid u$. The sub-bands are divided into $K$ clusters each containing $\frac{u}{K}$ sub-bands. Each cluster is assigned to a user. For example, if there is only one active user in the system, $\frac{K-1}{K} u$ frequency bands are unused. However, the FH scheme allows this active user to spread its power on the whole band achieving a higher spectral efficiency.

On the other hand, since the FD system is designed to handle the case where the number of active users is $K$, the minimum multiplexing gain per user is $\eta_{3}^{(F D)}=\frac{u}{K}$. As we will see, $\eta_{3}^{(F H)}=\frac{u}{K}\left(1-\frac{1}{K}\right)^{K-1}$ which is less than $\eta_{3}^{(F D)}$. However, one can easily observe that $\frac{\eta_{3}^{(F H)}}{\eta_{3}^{(F D)}} \geq \frac{1}{e}$ for all $K$, i.e., the loss incurred in the FH system in terms of $\eta_{3}$ is not more than $\frac{1}{e}$.

It might happen that there are more than $u$ users in the system. Clearly, the FD system is not capable to provide service for all of these users. On the other hand, the FH system allows all of these users to share the spectrum. This is interpreted as a higher service capability.

The paper outline is as follows. System model is given in section II. In section III, upper bounds on the achievable rates of users are computed. Section IV offers lower bounds on the achievable rates of users. In section $\mathrm{V}$, based on the results in sections III and IV, we discuss how the users in the FH system fairly share the band while maximizing the multiplexing gain per user. Comparison between the $\mathrm{FH}$ and FD systems is given in this section. We use the notation $f(\gamma) \sim g(\gamma)$ implying $\lim _{\gamma \rightarrow \infty} \frac{f(\gamma)}{g(\gamma)}=1$ throughout the paper. Because of space limitations, we refer the reader to [4] for the proof of proposition 1 and details on the diversity- multiplexing tradeoff.

\section{System Model}

We consider a communication system with $n$ users where the $i^{\text {th }}$ user exploits $v_{i}(\leq u)$ out of the $u$ sub-bands and spreads its available power, $P$, equally over these selected bands by transmitting independent Gaussian signals of variance $\frac{P}{v_{i}}$ over each of the chosen sub-bands. This user hops to another set of $v_{i}$ frequency sub-bands after each transmission. We denote the achievable rate of the $i^{t h}$ user by $R_{i}$. The static and non frequency-selective fading coefficient of the link connecting the $i^{\text {th }}$ transmitter to the $j^{\text {th }}$ receiver is shown by $h_{i, j}$. Each receiver knows already the hopping pattern of its affiliated transmitter. On the other hand, as all users hop over different portions of the spectrum from transmission to transmission, no user is assumed to be capable of tracking the instantaneous interference. This assumption makes the interference plus noise PDF at the receiver side of each user be a mixed Gaussian distribution. In fact, depending on different choices the other users make to select the frequency subbands and values of the crossover gains, this mixed Gaussian distribution has up to $2^{n-1}$ power levels. For each $i$, the channel model for the $i^{\text {th }}$ link is as follows:

$$
\vec{Y}_{i}=h_{i, i} \vec{X}_{i}+\vec{Z}_{i}
$$

where $\vec{X}_{i}$ is the $u \times 1$ input vector of the $i^{t h}$ user and $\vec{Z}_{i}$ is the noise plus interference vector on the receiver side of the $i^{\text {th }}$ user. One may write $p_{\vec{X}_{i}}(\vec{x})=\sum_{C \in \mathcal{C}} \frac{1}{\left(\begin{array}{l}u \\ v_{i}\end{array}\right)} g(\vec{x}, C)$ where $g(\vec{x}, C)$ denotes a zero-mean jointly Gaussian distribution of covariance matrix $C$ and the set $\mathcal{C}$ includes all $u \times u$ diagonal matrices where $v_{i}$ out of the $u$ diagonal elements are $\frac{P}{v_{i}}$ while the rest are zeros. Denoting the noise plus interference on the $j^{t h}$ band at the receiver side of the $i^{\text {th }}$ user by $Z_{i, j}$ (the $j^{\text {th }}$ component of $\vec{Z}_{i}$ ), it is clear that $p_{Z_{i, j}}(z)$ is not dependent on $j$. This is by the fact that crossover gains are not sensitive to frequency and there is no particular interest to a specific frequency sub-band by any user. We assume there are $L_{i}+1$ $\left(L_{i} \leq 2^{n-1}-1\right)$ possible non-zero power levels for $Z_{i, j}$, say $\left\{\sigma_{i, l}^{2}\right\}_{l=0}^{L_{i}}$. The occurrence probability of $\sigma_{i, l}^{2}$ is denoted by $a_{i, l}$. Then, $p_{Z_{i, j}}(z)$ is a mixed Gaussian distribution as follows:

$$
p_{Z_{i, j}}(z)=\sum_{l=0}^{L_{i}} \frac{a_{i, l}}{\sqrt{2 \pi} \sigma_{i, l}} \exp -\frac{z^{2}}{2 \sigma_{i, l}^{2}}
$$

where $\sigma^{2}=\sigma_{i, 0}^{2}<\sigma_{i, 1}^{2}<\sigma_{i, 2}^{2}<\ldots<\sigma_{i, L_{i}}^{2}\left(\sigma^{2}\right.$ is the ambient noise power). In fact, one may write $Z_{i, j}=$ $\sum_{k=1, k \neq i}^{n} \epsilon_{k, j} h_{k, i} X_{k, j}+\nu_{i, j}$ where $X_{k, j}$ is the signal of the $k^{t h}$ user sent on the $j^{t h}$ sub-band, $\epsilon_{k, j}$ is a Bernoulli random variable showing if the $k^{t h}$ user has utilized the $j^{t h}$ sub-band and $\nu_{i, j}$ is the ambient noise which is a zeromean Gaussian random variable with variance $\sigma^{2}$. Obviously, $\operatorname{Pr}\left\{\epsilon_{k, j}=1\right\}=\frac{v_{k}}{u}$. Also, a quantity of interest would be the following:

$$
a_{i, 0}=\operatorname{Pr}\left\{Z_{i, j} \text { contains no interference }\right\}
$$




$$
=\prod_{k \neq i} \operatorname{Pr}\left\{\epsilon_{k, j}=0\right\}=\prod_{k \neq i}\left(1-\frac{v_{i}}{u}\right) .
$$

We notice that for each $l \geq 1$, there exists a $c_{i, l}>0$ such that $\sigma_{i, l}^{2}=\sigma^{2}+c_{i, l} P$ where $c_{i, 1}<c_{i, 2}<\ldots<c_{i, L_{i}}$. To compute $R_{i}$, one may see that for each $i$, the communication channel of the $i^{t h}$ user is a channel with state $S_{i}$, the hopping pattern, which is independently changing over different transmissions and is known to both the transmitter and receiver ends of the $i^{t h}$ user. The achievable rate of such a channel is given by

$$
R_{i}=I\left(\vec{X}_{i} ; \vec{Y}_{i} \mid S_{i}\right)=\sum_{s_{i} \in \mathcal{S}_{i}} \operatorname{Pr}\left(S_{i}=s_{i}\right) I\left(\vec{X}_{i} ; \vec{Y}_{i} \mid S_{i}=s_{i}\right)
$$

where $I\left(\vec{X}_{i} ; \vec{Y}_{i} \mid S_{i}=s_{i}\right)$ is the mutual information between $\vec{X}_{i}$ and $\vec{Y}_{i}$ for the specific sub-band selection dictated by $S_{i}=s_{i}$. The set $\mathcal{S}_{i}$ denotes all possible selections of $v_{i}$ out of the $u$ subbands. As $p_{\vec{Z}_{i}}(\vec{z})$ is a symmetric density function, meaning all its components have the same PDF given in (2), we deduce that $I\left(\vec{X}_{i} ; \vec{Y}_{i} \mid S_{i}=s_{i}\right)$ is independent of $s_{i}$. Therefore, we may assume any specific sub-band selection for the $i^{\text {th }}$ user in $\mathcal{S}_{i}$, say the first $v_{i}$ out of the $u$ sub-bands. Denoting this specific state by $s_{i}^{*}$, we get:

$$
R_{i}=I\left(\vec{X}_{i} ; \vec{Y}_{i} \mid S_{i}=s_{i}^{*}\right) .
$$

In this case, we denote $\vec{Y}_{i}$ and $\vec{X}_{i}$ by $\vec{Y}_{i}\left(s_{i}^{*}\right)$ and $\vec{X}_{i}\left(s_{i}^{*}\right)$ respectively. Obviously, we have:

$$
R_{i}=I\left(\vec{X}_{i}\left(s_{i}^{*}\right) ; \vec{Y}_{i}\left(s_{i}^{*}\right)\right)=\mathrm{h}\left(\vec{Y}_{i}\left(s_{i}^{*}\right)\right)-\mathrm{h}\left(\vec{Z}_{i}\right) .
$$

Throughout the paper, the number of users is assumed to be a random variable. To decode the data, the receiver of the $i^{t h}$ user is expected to know the noise plus interference PDF, $p_{\vec{Z}_{i}}(\vec{z})$. As we will see, each transmitter can regulate its rate close to its achievable rate within a gap which is bounded in terms of SNR. To do this, each transmitter only needs to know the greatest interference level on each frequency sub-band at the receiver side and its forward channel gain. Clearly, if the gains $\left\{h_{i, j}\right\}$ have a continuous distribution, the number of interference levels is equal to $2^{n-1}$ with probability one. As such, $n$ is also assumed to be a global knowledge among users.

\section{UPPER Bounds on THE ACHIEVABle RATES}

Let $\vec{W}_{i}$ be the $u \times 1$ interference vector at the receiver side of the $i^{\text {th }}$ user where its $j^{\text {th }}$ component, $W_{i, j}$, is a random variable showing the interference term on the $j^{\text {th }}$ frequency sub-band. In terms of our previous notation, $W_{i, j}=\sum_{k=1, k \neq i}^{n} \epsilon_{k, j} h_{k, i} X_{k, j}$. We have $p_{\vec{W}_{i}}(\vec{w})=$ $\frac{1}{M_{i}} \sum_{m=1}^{M_{i}} g\left(\vec{w}, D_{i, m}\right)$ where $M_{i}=\prod_{j \neq i}\left(\begin{array}{c}u \\ v_{j}\end{array}\right)$ and as each user transmits independent Gaussian signals through its chosen sub-bands, the matrices $\left\{D_{i, m}\right\}_{m=1}^{M_{i}}$ are diagonal, i.e., $D_{i, m}=\operatorname{diag}\left(d_{i, m}^{(1)}, \cdots, d_{i, m}^{(u)}\right)$. If the PDF of the interference vector consisted only of $g\left(\vec{w}, D_{i, m}\right)$, the forward link of the $i^{\text {th }}$ channel would be converted to an additive Gaussian noise channel. The achievable rate of such a virtual channel is simply given by:

$$
\begin{aligned}
R_{i, m} & =\frac{1}{2} \log \frac{\operatorname{det}\left(\operatorname{Cov}\left(\vec{X}_{i}\left(s_{i}^{*}\right)\right)+D_{i, m}+\sigma^{2} I_{u}\right)}{\operatorname{det}\left(D_{i, m}+\sigma^{2} I_{u}\right)} \\
& =\frac{1}{2} \sum_{j=1}^{v_{i}} \log \left(1+\frac{\left|h_{i, i}\right|^{2} P}{v_{i}\left(d_{i, m}^{(j)}+\sigma^{2}\right)}\right) .
\end{aligned}
$$

Let $T_{i, m}=\left\{j \mid 1 \leq j \leq v_{i}, d_{i, m}^{(j)}=0\right\}$. Defining $\gamma=\frac{P}{\sigma^{2}}$, we get:

$$
R_{i, m}=\frac{\left|T_{i, m}\right|}{2} \log \left(1+\frac{\left|h_{i, i}\right|^{2} \gamma}{v_{i}}\right)+\tilde{R}_{i, m}
$$

where $\tilde{R}_{i, m}=\frac{1}{2} \sum_{1 \leq j \leq v_{i}: d_{i, m}^{(j)} \neq 0} \log \left(1+\frac{\left|h_{i, i}\right|^{2} P}{v_{i}\left(d_{i, m}^{(j)}+\sigma^{2}\right)}\right)$. As each non-zero $d_{i, m}^{(j)}$ is proportional to $P$, it is clear that $\lim _{\gamma \rightarrow \infty} \tilde{R}_{i, m}<\infty$. On the other hand, $R_{i}$ is convex in terms of $p_{\vec{Y}_{i}\left(s_{i}^{*}\right) \mid \vec{X}_{i}\left(s_{i}^{*}\right)}(\vec{y} \mid \vec{x})=p_{\vec{Z}_{i}}(\vec{y}-\vec{x})$. But, $p_{\vec{Z}_{i}}(\vec{z})=$ $\frac{1}{M_{i}} \sum_{m=1}^{M_{i}} g\left(\vec{z}, D_{i, m}+\sigma^{2} I_{u}\right)$. Therefore, we have:

$$
\begin{gathered}
R_{i} \leq \frac{1}{M_{i}} \sum_{m=1}^{M_{i}} R_{i, m} \\
=\frac{1}{2}\left(\frac{1}{M_{i}} \sum_{m=1}^{M_{i}}\left|T_{i, m}\right|\right) \log \left(1+\frac{\left|h_{i, i}\right|^{2} \gamma}{v_{i}}\right)+\tilde{R}_{i}
\end{gathered}
$$

where $\tilde{R}_{i}=\frac{1}{M_{i}} \sum_{m=1}^{M_{i}} \tilde{R}_{i, m}$. As each $\tilde{R}_{i, m}$ saturates by increasing $\gamma$, one has $\lim _{\gamma \rightarrow \infty} \tilde{R}_{i}<\infty$. The following lemma offers an explicit expression for $\frac{1}{M_{i}} \sum_{m=1}^{M_{i}}\left|T_{i, m}\right|$.

\section{Lemma 1}

$$
\frac{1}{M_{i}} \sum_{m=1}^{M_{i}}\left|T_{i, m}\right|=v_{i} \prod_{k=1, k \neq i}^{n}\left(1-\frac{v_{k}}{u}\right) .
$$

Proof: Defining $A_{j}=\left\{m:\left|T_{i, m}\right|=j\right\}$, one may express the left side as $\frac{1}{M_{i}} \sum_{m=1}^{M_{i}}\left|T_{i, m}\right|=\frac{1}{M_{i}} \sum_{j=1}^{v_{i}} j\left|A_{j}\right|$. Let $F$ be a random variable showing the number of interferencefree sub-bands among the $v_{i}$ sub-bands selected by the $i^{\text {th }}$ user. Noting that $\operatorname{Pr}\{F=j\}=\frac{\left|A_{j}\right|}{M_{i}}$, we have $\frac{1}{M_{i}} \sum_{m=1}^{M_{i}}\left|T_{i, m}\right|=\sum_{j=1}^{v_{i}} j \operatorname{Pr}\{F=j\}=E\{F\}$. Let us define $F_{j}=\left\{\begin{array}{ll}1 & W_{i, j}=0 \\ 0 & W_{i, j} \neq 0\end{array}\right.$. Obviously, $F=\sum_{j=1}^{v_{i}} F_{j}$. As such, we get $E\{F\}=\sum_{j=1}^{v_{i}} E\left\{F_{j}\right\}=\sum_{j=1}^{v_{i}} \operatorname{Pr}\left\{W_{i, j}=0\right\}$. But, $\forall j: \operatorname{Pr}\left\{W_{i, j}=0\right\}=a_{i, 0}=\prod_{k=1, k \neq i}^{n}\left(1-\frac{v_{k}}{u}\right)$ which yields $E\{F\}=v_{i} \prod_{k=1, k \neq i}^{n}\left(1-\frac{v_{k}}{u}\right)$.

Based on (9) and lemma 1, we propose the following theorem:

Theorem 1 There exists an upper bound $R_{i}^{u b}$ on the achievable rate of the $i^{\text {th }}$ user which satisfies

$$
R_{i}^{u b} \sim \frac{1}{2} v_{i} \prod_{k=1, k \neq i}^{n}\left(1-\frac{v_{k}}{u}\right) \log \gamma .
$$

\section{LOWER BOUNDS ON THE RATES}

We define $\vec{X}_{i}^{\prime}$ to be the $v_{i} \times 1$ signal vector corresponding to the first $v_{i}$ elements of $\vec{X}_{i}\left(s_{i}^{*}\right)$. Let $\vec{Y}_{i}^{\prime}=h_{i, i} \vec{X}_{i}^{\prime}+\vec{Z}_{i}^{\prime}$ where 
$\vec{Z}_{i}^{\prime}$ is the noise plus interference vector at the receiver side on the first $v_{i}$ sub-bands. By entropy power inequality, we have:

$$
2^{\frac{2}{v_{i}} \mathrm{~h}\left(\vec{Y}_{i}^{\prime}\right)} \geq 2^{\frac{2}{v_{i}} \mathrm{~h}\left(h_{i, i} \vec{X}_{i}^{\prime}\right)}+2^{\frac{2}{v_{i}} \mathrm{~h}\left(\vec{Z}_{i}^{\prime}\right)} .
$$

Dividing both sides by $2^{\mathrm{h}\left(\vec{Z}_{i}^{\prime}\right)}$, we get:

$$
\mathrm{h}\left(\vec{Y}_{i}^{\prime}\right)-\mathrm{h}\left(\vec{Z}_{i}^{\prime}\right) \geq \frac{v_{i}}{2} \log \left(2^{\frac{2}{v_{i}}\left(\mathrm{~h}\left(h_{i, i} \vec{X}_{i}^{\prime}\right)-\mathrm{h}\left(\vec{Z}_{i}^{\prime}\right)\right)}+1\right) .
$$

On the other hand, since $\vec{Y}_{i}^{\prime}$ is a subvector of $\vec{Y}_{i}\left(s_{i}^{*}\right)$, we have:

$$
R_{i}=I\left(\vec{X}_{i}\left(s_{i}^{*}\right) ; \vec{Y}_{i}\left(s_{i}^{*}\right)\right) \geq I\left(\vec{X}_{i}^{\prime} ; \vec{Y}_{i}^{\prime}\right)=\mathrm{h}\left(\vec{Y}_{i}^{\prime}\right)-\mathrm{h}\left(\vec{Z}_{i}^{\prime}\right) .
$$

Comparing (11) and (12) yields:

$$
R_{i} \geq \frac{v_{i}}{2} \log \left(2^{\frac{2}{v_{i}}\left(\mathrm{~h}\left(h_{i, i} \vec{X}_{i}^{\prime}\right)-\mathrm{h}\left(\vec{Z}_{i}^{\prime}\right)\right)}+1\right) .
$$

Clearly, $\mathrm{h}\left(h_{i, i} \vec{X}_{i}^{\prime}\right)=\frac{v_{i}}{2} \log \left(2 \pi e \frac{\left|h_{i, i}\right|^{2} P}{v_{i}}\right)$. As $\vec{Z}_{i}^{\prime}$ is a mixed Gaussian vector, there is no closed-form formula for $\mathrm{h}\left(\vec{Z}_{i}^{\prime}\right)$. Hence, we have to find an appropriate upper bound on $\mathrm{h}\left(\vec{Z}_{i}^{\prime}\right)$. Using the chain rule for the differential entropy yields:

$$
\mathrm{h}\left(\vec{Z}_{i}^{\prime}\right) \leq \sum_{j=1}^{v_{i}} \mathrm{~h}\left(Z_{i, j}\right)
$$

The following proposition whose proof is offered in [4] yields an upper bound on $\mathrm{h}\left(Z_{i, j}\right)$.

Proposition 1 For every $1 \leq j \leq v_{i}$ and for all values of $\gamma$, there exists an upper bound on $\mathrm{h}\left(Z_{i, j}\right)$ given by

$$
\mathrm{h}\left(Z_{i, j}\right) \leq \frac{1}{2}\left(1-a_{i, 0}\right) \log \left(c_{i, L_{i}} \gamma+1\right)+\log (\sqrt{2 \pi e} \sigma)+\kappa_{i}
$$

where $\kappa_{i}=\mathcal{H}\left(a_{i, 0}, \cdots, a_{i, L_{i}}\right)$ is the discrete entropy of $\left\{a_{i, j}\right\}_{j=0}^{L_{i}}$.

Based on proposition 1, and by (13) and (14), we get:

$$
R_{i} \geq R_{i}^{l b}:=\frac{v_{i}}{2} \log \left(\frac{2^{-2 \kappa_{i}}\left|h_{i, i}\right|^{2} \gamma}{v_{i}\left(c_{i, L_{i}} \gamma+1\right)^{1-a_{i, 0}}}+1\right) .
$$

But, $\frac{v_{i}}{2} \log \left(\frac{2^{-2 \kappa_{i}}\left|h_{i, i}\right|^{2} \gamma}{v_{i}\left(c_{i, L_{i}} \gamma+1\right)^{1-a_{i, 0}}}+1\right) \sim \frac{1}{2} v_{i} a_{i, 0} \log \gamma$. Thus, we come up with the following result of this section:

Theorem 2 There exists a lower bound $R_{i}^{l b}$ on the achievable rate of the $i^{\text {th }}$ user which satisfies

$$
R_{i}^{l b} \sim \frac{1}{2} v_{i} \prod_{k=1, k \neq i}^{n}\left(1-\frac{v_{k}}{u}\right) \log \gamma .
$$

From now on, we assume that the $i^{\text {th }}$ transmitter regulates its rate at $R_{i}^{l b}$. It can be seen that the only parameters needed to compute $R_{i}^{l b}$ are $\left|h_{i, i}\right|, c_{i, L_{i}}$ and $\kappa_{i} . c_{i, L_{i}}$ represents the greatest interference level on each sub-band at the receiver of the $i^{t h}$ user. This together with $\left|h_{i, i}\right|$ must be passed over to the transmitter side via a feedback link.

\section{System DESIGN}

In this section, we consider the complex case where signals, ambient noise and channel gains are circular complex Gaussian random variables. This affects the previous results via multiplication by a factor of two. In general, there are two fixed parameters in the system, the number of frequency bands, $u$, and the maximum number of active users that the system is designed to handle, $K$. We compare the FH system with the FD system according to four key measures to be defined later. Based on the results in the previous sections, there exist upper and lower bounds on the achievable rate of each user which coincide in the high SNR regime. Thus, the achievable rate itself must be asymptotically equivalent to each of these bounds, i.e.,

$$
R_{i} \sim v_{i} \prod_{k=1, k \neq i}^{n}\left(1-\frac{v_{k}}{u}\right) \log \gamma .
$$

Let $S R=\sum_{i=1}^{n} R_{i}$ be the sum-rate. Then,

$$
S R \sim r_{S R} \log \gamma
$$

where

$$
r_{S R}=\sum_{i=1}^{n} v_{i} \prod_{1 \leq k \leq n, k \neq i}\left(1-\frac{v_{k}}{u}\right) .
$$

We call $r_{S R}$ the sum-rate multiplexing gain of the system. $r_{S R}$ is a symmetric function of $v_{i}$ 's. In a "fair" FH system, it is required that $v_{i}=v$ for all $i$. Thus,

$$
r_{S R}=n v\left(1-\frac{v}{u}\right)^{n-1} \text {. }
$$

Maximizing this in terms of $v$ yields:

$$
v_{\text {opt }}=\left\{\begin{array}{cl}
1 & \text { if } \frac{u}{n}<1 \\
\left\lfloor\frac{u}{n}\right\rfloor & \text { if } \frac{u}{n} \geq 1
\end{array} .\right.
$$

In the sequel, we compare the performance of the FD system with that of the decentralized network adopting the FH strategy. We assume the number of users in the system is a random variable $N$ with probability mass function $q_{n}=\operatorname{Pr}\{N=n\}$ for $n \geq 1$. For the moment, we assume $\operatorname{Pr}\{N>K\}=0$. In what follows all expectations are with respect to the number of active users. Our comparison is based on four performance measures namely, average sum-rate multiplexing gain, average multiplexing gain per user, the minimum multiplexing gain per user and the so-called service capability. Service capability shows the fraction of users getting service among all the active users in the system. For brevity, we take $K=2$ in all the examples provided. For more general examples, we refer the reader to [4].

- Average sum-rate multiplexing gain

Average sum-rate multiplexing gain is defined as $\eta_{1}=$ $E\left\{r_{S R}\right\}$. The FD system is already designed to handle $K \leq u$ users where $K \mid u$. The frequency sub-bands are divided into $K$ clusters each containing $\frac{u}{K}$ sub-bands. Each user that enters the system looks for an empty cluster. If there is one, the user occupies the cluster. If there is no empty cluster, no service is 
available. Therefore, the sum-rate multiplexing gain is

$$
r_{S R}^{(F D)}=\left\{\begin{array}{cc}
N \frac{u}{K} & N \leq K \\
u & N>K
\end{array} .\right.
$$

On the other hand, in a decentralized network with $\mathrm{FH}$ strategy, the parameter $K$ is meaningless. In fact, by the nature of FH, any number of active users can get service. Since $N$ is a global knowledge, by (19) and (20), $r_{S R}^{(F H)}$ is given by:

$$
r_{S R}^{(F H)}=\left\{\begin{array}{cc}
N\left\lfloor\frac{u}{N}\right\rfloor\left(1-\frac{1}{u}\left\lfloor\frac{u}{N}\right\rfloor\right)^{N-1} & N \leq u \\
N\left(1-\frac{1}{u}\right)^{N-1} & N>u
\end{array}\right.
$$

Example 1 Assume there are always at most two active users in the system and $2 \mid u$. As such, the central controller in the FD system sets $K=2$, and according to (21), we have $\eta_{1}^{(F D)}=$ $E\left\{r_{S R}^{(F D)}\right\}=q_{1} \frac{u}{2}+q_{2} u$. On the other hand, based on (22), we get $\eta_{1}^{(F H)}=E\left\{r_{S R}^{(F H)}\right\}=q_{1} u+2 q_{2} \frac{u}{2}\left(1-\frac{1}{u} \frac{u}{2}\right)=q_{1} u+q_{2} \frac{u}{2}$. Therefore, as far as $q_{1} u+q_{2} \frac{u}{2}>q_{1} \frac{u}{2}+q_{2} u$ or equivalently $q_{1}>q_{2}$, we have $\eta_{1}^{(F H)}>\eta_{1}^{(F D)}$. Thus, if $q_{1}>\frac{1}{2}$, i.e., the probability that two users become active simultaneously is less than $\frac{1}{2}$, the FH system utilizes the band more efficiently.

- Average multiplexing gain per user

Average multiplexing gain per user is defined as $\eta_{2}=$ $E\left\{\frac{r_{S R}}{N}\right\}$. This measure shows the multiplexing gain each user achieves on average.

Example 2 Considering the same setup as in example 1, we have $\eta_{2}^{(F D)}=\frac{u}{2}$ and $\eta_{2}^{(F H)}=q_{1} u+q_{2} \frac{u}{2}\left(1-\frac{1}{u} \frac{u}{2}\right)=$ $q_{1} u+q_{2} \frac{u}{4}$. Therefore, as far as $q_{1} u+q_{2} \frac{u}{4}>\frac{u}{2}$ or equivalently $q_{1}>\frac{1}{3}$, we have $\eta_{2}^{(F H)}>\eta_{2}^{(F D)}$. This example together with example 1 show that as far as $q_{1}>\frac{1}{2}$, the FH system outperforms the FD system in terms of both $\eta_{1}$ and $\eta_{2}$.

- Minimum multiplexing gain per user

The minimum multiplexing gain per user is the smallest possible multiplexing gain that a user attains. We denote this by $\eta_{3}$. Clearly, this happens when there are exactly $K$ active users in the system. As the FD system is already designed to handle the case where $K$ users are present in the system, the minimum multiplexing gain per user is automatically higher. Setting $N=K$, we have $\eta_{3}^{(F D)}=\frac{r_{S R}^{(F D)}}{K}=\frac{u}{K}$ and $\eta_{3}^{(F H)}=$ $\frac{r_{S R}^{(F H)}}{K}=\frac{u}{K}\left(1-\frac{1}{K}\right)^{K-1}$ by (21) and (22) respectively. Clearly, $\frac{1}{e} \leq \frac{\eta_{3}^{(F H)}}{\eta_{3}^{(F D)}} \leq 1$ as $\left(1-\frac{1}{K}\right)^{K-1}$ approaches $\frac{1}{e}$ from above by increasing $K$. Therefore, the loss incurred in the FH system is at most $\frac{1}{e}$.

- Service capability

Service capability demonstrates the fraction of users getting service out of the whole present users in the system. Let us denote the number of users getting service by $N_{s}$. Therefore, the service capability is computed as $E\left\{\frac{N_{s}}{N}\right\}$. In the FH system, the service capability is always one. But, in the FD system, if $N>u$ then certainly a fraction of users can not share the band. This actually occurs whenever $\operatorname{Pr}\{N>u\}>0$. In case $\operatorname{Pr}\{N \leq u\}=1$, both systems have service capability equal to one.

\section{REFERENCES}

[1] R. Etkin, A.P. Parekh and D. Tse "Spectrum sharing in unlicensed bands", IEEE Journal on Selected Areas in Communications, vol. 25, no. 3, pp. 517-528, April 2007.

[2] J. Mitola, "Cognitive radio: an integrated agent architecture for software defined radio", PhD Dissertation, KTH, Stockholm, Sweden, Dec. 2000.

[3] N. Devroye, P. Mitran and V. Tarokh, "Achievable rates in cognitive radio channels", IEEE Trans. Inf. Theory, vol. 52, no. 5, pp. 1813-1827, May 2006.

[4] K Moshksar, A. Bayesteh and A.K. Khandani, "Spectrum Sharing vs Spectral Efficiency in Decentralized Networks", Tech. Report, Available Online at http://shannon2.uwaterloo.ca/ kmoshksa/ 